\title{
AN IMPLANTABLE INTEGRATED SIGE FM TRANSMITTER FOR HRV BIOTELEMETRY
}

\author{
Fang-Ren Liao, Chi-An Chen, Shey-Shi Lu, Nan-Fu Chiu*, Chii-Wann Lin*, Jen-Yu Li*, \\ Chia-Nan Chien**, Fu-Shan Jaw**, Jiun-Min Wang ${ }^{+}$,Lung-Jieh Yang ${ }^{+}$, Tzu-Chien Hsiao ${ }^{++}$ \\ and Chih-Kung Lee
}
Graduate Institute of Electronics Engineering and Department of Electrical Engineering, National Taiwan University, Taiwan, ROC
*Department of Electrical Engineering, National Taiwan University, Taiwan, ROC
** Institute of Biomedical Engineering, National Taiwan University, Taiwan, ROC
${ }^{+}$Department of Mechanical and Electro-Mechanical Engineering, Tamkang University
"Department of Biomedical Engineering, College of Medicine, I-Shou University
Institute of Applied Mechanics, National Taiwan University, Taiwan, ROC

\section{ABSTRACT}

This paper presents an implantable integrated FM transmitter using $0.35 \mathrm{um}$ SiGe process for biotelemetry. The arehitecture of FM transmitter is based on the Colpitts oscillater, which is followed by a buffer amplifier to isolate the influence of output on the resonant frequency of the oscillator. The chip area of the fabricated FM transmitter is only $0.26 \mathrm{~mm}^{2}$. Even without an intentional antenna, the experimental results show that electrocardiograms (ECGs) of a rat using the FM transmitter can be successfully transmitted to a personal computer, where the heart rate variability (HRV) is analyzed.

\section{INTRODUCTION}

Recently, as the development of system-on-chip (SoC) technologies advances, there has been a growing interest in implantable wireless biotelemetry for diagnosis [1]-[5]. Many of them are based on the digital communication process $[1][3][4][5]$, i.e. the measured signals from biosensors are first processed by analog-to-digital converter (ADC) and then transmitted to receivers by amplitude- or frequency-shifted key-in (ASK, FSK) techniques. As a result, the whole system becomes complex and the die size is too large to be implanted inside the human body. In fact, some biomedical parameters for diagnosis, such as heart rate variability (HRV), do not require high-fidelity lincar transmission. Taking the HRV for example, only the time intervals between the heart-beats (so called R-R intervals) are important. Under such a circumstance, a simple transmitter with a small die size is sufficient. In this paper, we report the implementation of a miniaturized integrated FM transmitter for transmitting the electrocardiograms (ECGs) of a rat to a personal computer, where the heart rate variability (HRV) is analyzed by Fourier transform. The transmitter is operated at $1.6 \mathrm{~V}$ with a current of $3 \mathrm{~mA}$. Transmitting frequency is about 96 MHz. The received ECG signals of the rat in the PC are processed real-time by LabView for diagnosis.

\section{CIRCUIT DESIGN}

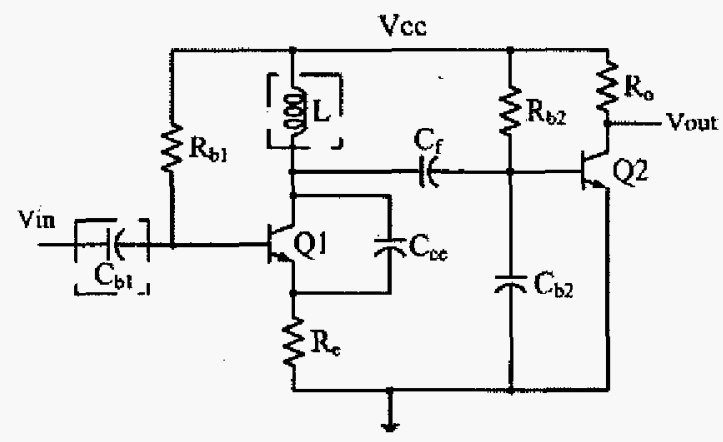

Fig. 1 The schematic of the FM transmitter. Off-chip components are indicated by their circuit symbols enclosed in dash boxes.

The core of the FM transmitter is the Colpitts oscillator (see Fig. 1) composed of $\mathrm{Ql}$ (emitter size $20.3 \mathrm{um} \times 0.3 \mathrm{um}), \mathrm{L}(0.82 \mathrm{uH}), \mathrm{C}_{\mathrm{ee}}(5.0 \mathrm{pF})$ and $R_{\mathrm{e}}$ $(200 \Omega)$. The resistors $R_{b 1}(62 \mathrm{k} \Omega)$ and $R_{b 2}(47 \mathrm{k} \Omega)$ are for biasing and the capacitor $C_{b 1}(1.0 \mathrm{uF})$ is for AC coupling. The output of the Colpitts oscillator is followed by a buffer amplifier consisting of a transistor $\mathrm{Q} 2$ (emitter size $20.3 \mathrm{um} \times 0.3 \mathrm{um}$ ), $\mathrm{C}_{\mathrm{b} 2}$ $(33.0 \mathrm{pF})$ and a load resistor $R_{0}(200 \Omega)$ to isolate the influence of the transmitter output (Vout) on the resonant frequency of the osciliator [6]. A large inductance value $(0.82 \mathrm{uH})$ is required because of the low transmitting frequency chosen ( $96 \mathrm{MHz})$. Since typical inductances provided by commercial foundries are around $\mathrm{nH}$, an off-chip surface mount inductor device (SMD) is used as indicated by the dash box in Fig. 1. Note that it is possible to integrate this inductor into the chip if a higher transmitting frequency is chosen. Since biomedical parameters are low frequency in nature, a large AC coupling capacitance $C_{b l}(1.0 \mathrm{uF})$ is needed and hence a surface mount capacitor device is used.

The FM transmitter was implemented in the TSMC 0.35um SiGe process. The die photograph of the finished circuit is shown in Fig. 2. The chip area 
is only $0.57 \mathrm{~mm} \times 0.46 \mathrm{~mm}$ including bonding pads. The transmitter chip is further wire-bonded to a printed circuit board (PCB) on which the two above-mentioned SMDs are soldered (see Fig. 3). Pitch is used to cover the chip for protection and the total size of the transmitter is about the size of a rice grain as is evidenced in Fig. 3. Note that no intentional antenna is used.

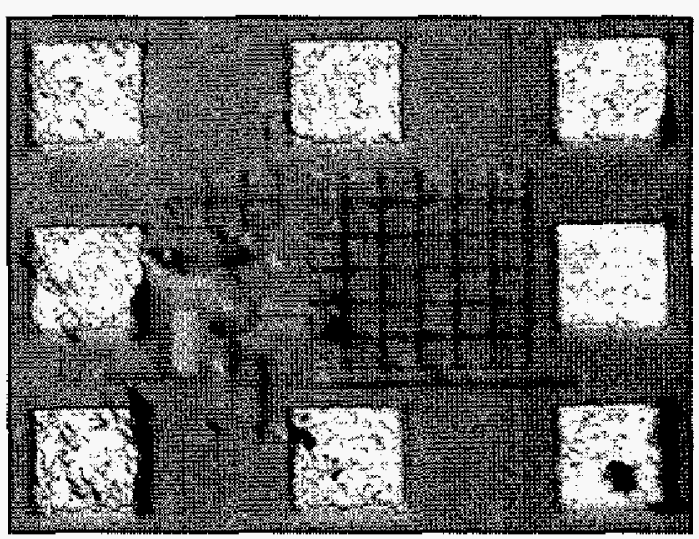

Fig. 2 The die photograph of the circuit.

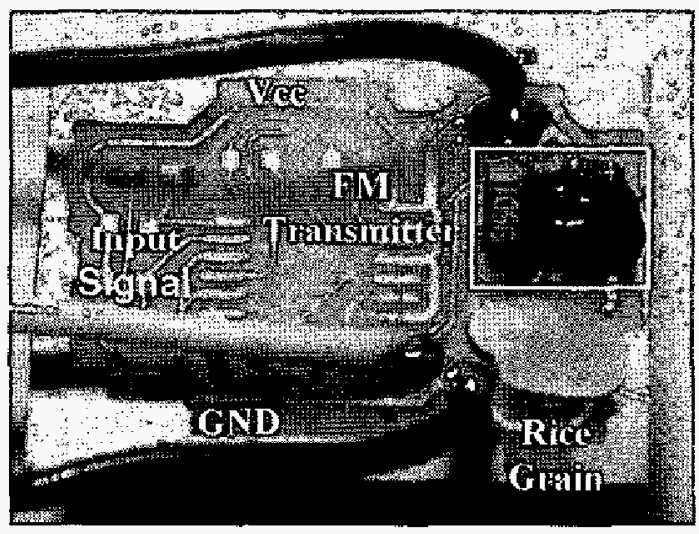

Fig. 3 The miniaturized FM transmitter. It is abont the same size as a rice grain.

\section{EXPERIMENT FOR HEART RATE VARIABILITY TEST}

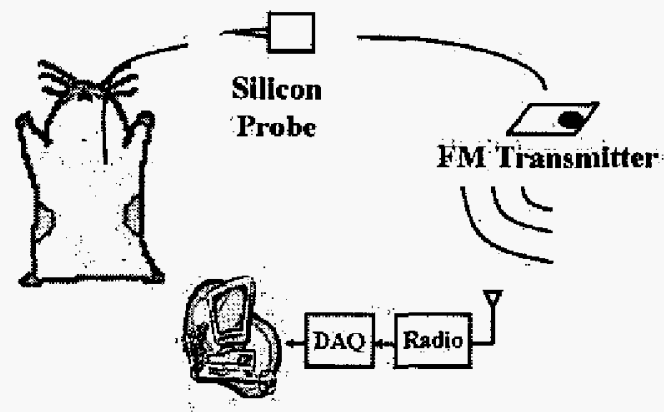

Fig. 4 Experimental set-up.
The transmitter is operated at $1.6 \mathrm{~V}$ with a current of 3 $m A$. In order to demonstrate that our FM transmitter can be used for biotelemetry, especially for wireless HRV analysis, the experimental set-up as illustrated in Fig. 4 is carried out. First a rat is anaesthetized. A silicon probe [7][8] (see Fig. 5) with four electrodes (working, counter, reference and physical-sensor electrodes) fabricated by MEMS process is used to take the ECG signals out from the rat. The miniaturized FM transmitter is then connected to the silicon probe for the transmission of ECG signals. The transmitted signals are received by a radio followed by a data acquisition (DAQ) card, which converts the analog data into digital form for the ease of data processing by Labview in the PC for HRV analysis. The received ECGs of the rat and the results after corresponding HRV analysis are shown in Fig. 6. The received ECGs were shown in section A of Fig. 6 where the features of $P, Q \cdot R \cdot S$ and $T$ of $E C G$ can be clearly identified. In section $B$ of Fig. 6 , the $R-R$ intervals of the rat are displayed. The results of HRV analysis are shown in section $C$ and $D$. The spectrum of the R-R interval by Fourier analysis is obtained in section $C$ while the histogram of R-R intervals with its associated mean and standard deviation are shown in section $D$. All these are important values for heart disease diagnosis. Another analysis method by Autoregressive (AR) modeling and the discussion for HRV can be seen in [9].

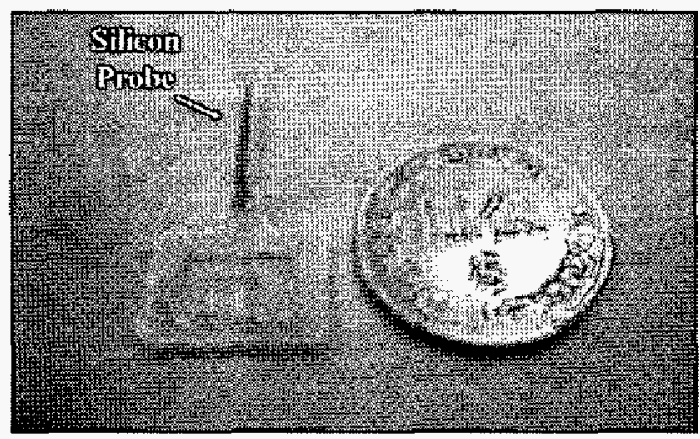

Fig. 5 The silicon probe used to take the ECG signals out from a rat.

\section{CONCLUSION}

In this paper, an integrated $\mathrm{SiGe}$ FM transmitter for wireless transmission of heart-rate-variability is presented. The fabricated transmitter is as small as a rice grain and hence is suitable for implantation purpose. The experimental results show that the ECG signals taken out fron a rat by a silicon probe can be transmitted and received wirelessly and successfully by the proposed miniaturized FM transmitter for HRV analysis. 


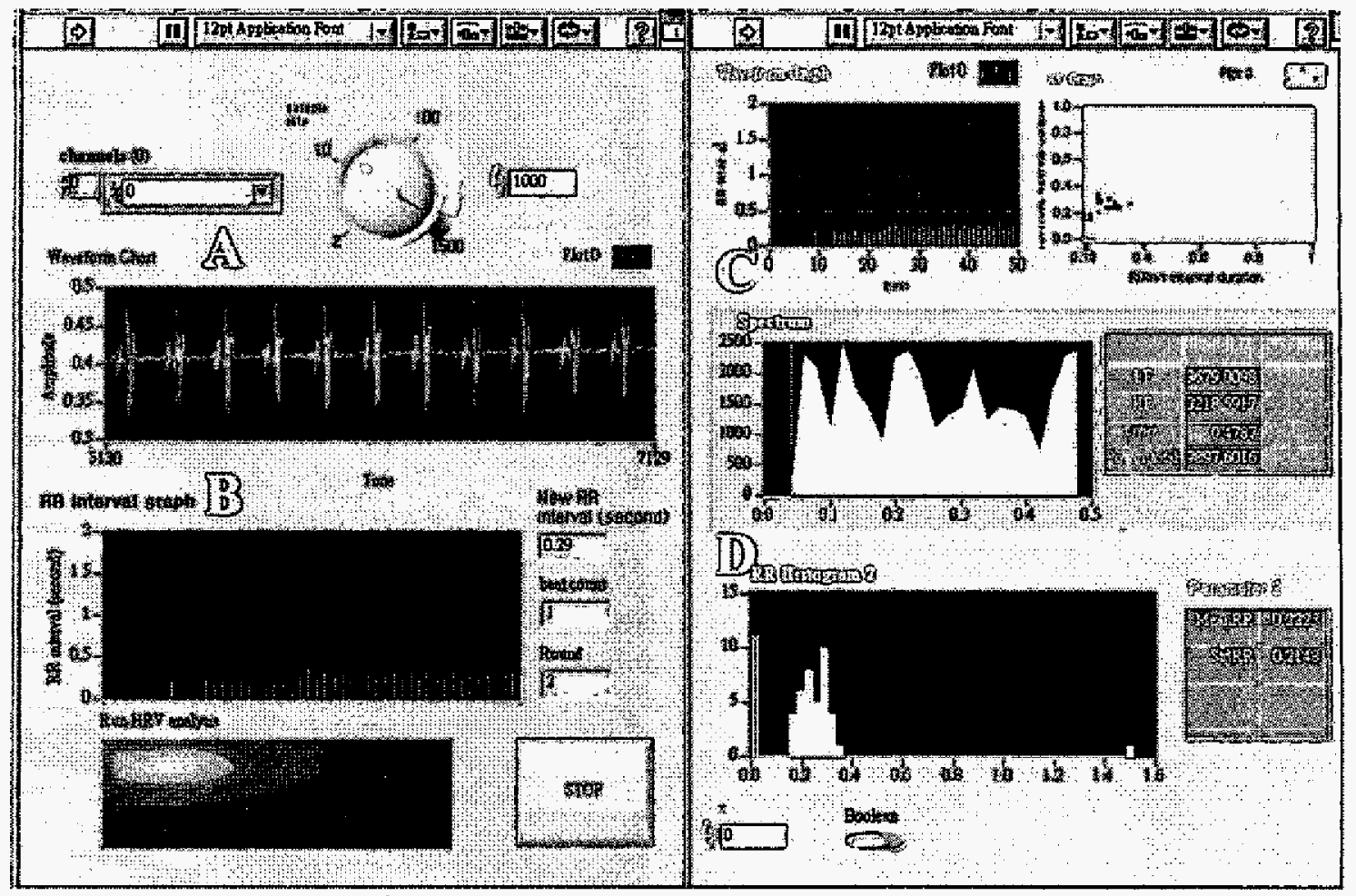

Fig. 6 The LabView program for HRV analysis. Section A: received ECG signals; section B: R-R intervals; section C: Fourier spectrum of R-R intervals; section D: histogram of R-R intervals with its associated mean and deviation.

\section{ACKNOWLEDGEMENT}

Financial and technical supports from Ministry of Economic Affairs under the contract 91EC17A05-S10017, NSC 93-2212-E-002-079 and $\mathrm{CIC}$ are appreciated.

\section{REFERENCES}

[1] Y. Kim and J. M. Cho, "Development of Wireless Bio-telemetry System Using FM Stereo Method for Exercising 'Rehabilitation Patients," in Proc. $23^{\text {rd }}$ Annual International Conference of IEEE EMBS, pp. 3338-3340, 2001.

[2] M. Ahmadian, B. W. Flynn, A. F. Murray and D. R. S. Cumming, "Miniature Transmitter for Implantable Micro Systems," in Proc. $25^{\text {th }}$ Annual International Conference of IEEE EMBS, pp. 3028-3031, 2003.

[3] N. Aydin, A. Astaras, L. Wang, T. Arslan, A. F. Murray, S. P. Beamont and D. R. S. Cumming, "Design and Implementation Considerations for an Advanced Wireless Interface in -Miniaturized Integrated Sensor Microsystems," in Proc. $25^{\text {th }}$ Anmual International Conference of IEEE EMBS, pp. 3400-3402, 2003.

[4] G. A. DelMichele and P. R. Troyk, "Tntegrated Multi-Channel Wireless Biotelemetry System," in Proc. $25^{\text {th }}$ Annual International Conference of IEEE EMBS, pp. 3372-3375, 2003.
[5] H. Yu and K. Najafi, "Low-Power Interface Circuits for Bio-Implantable Microsystems," IEEE International Solid-State Circuits Conference, pp. 194, February 2003.

[6] N. Ando, I. Shimoyama and R. Kanzaki, "A dual channel FM transmitter for acquisition of flight muscle activities from the freely flying hawkmoth, Agrius Convolvuli," Joumal of Neuroscience Methods, vol. 115, pp. 181-187, April 2002.

[7] L. Lin and A. P. Pisano, "Silicon-Processed Microneedles," IEEE Journal of Microelectromechanical Systems, vol. 8, pp. 78-84, March 1999.

[8] J. Brazzle, D. Bartholomeusz, R. Davies and J. Andrake, R. A. Van Wagenen, A. B. Frazier, "Active Microneedles With Integrated Functionality" Solid State Sensors and Actwators Conference, Hilton Head, 2000.

[9] H. G. Adelmann, "Design of a PC-Based System for Time-Domain and Spectral Analysis of Heart Rate Variability," Computers and Biomedical Research, vol. 32, pp 77-92, February 1999. 